\title{
Single range localization in 3D: observability and robustness issues
}

\author{
Giovanni Indiveri, Daniela De Palma and Gianfranco Parlangeli
}

\begin{abstract}
The issue of single range based observability analysis and observer design for the kinematics model of a 3D vehicle eventually subject to a constant unknown drift velocity is addressed. The proposed method departs from alternative solutions to the problem and leads to the definition of a linear time invariant state equation with a linear time varying output. Simple necessary and sufficient observability conditions are derived. The localization problem is finally solved using a novel outlier robust predictor - corrector state estimator. Numerical simulation examples are described to illustrate the performance of the method as compared to a standard Kalman filter.
\end{abstract}

\section{INTRODUCTION}

The problem of single range based localization is relevant in several land [1], [2], arial [3] and marine robotics [4] [5] application. In essence, the problem consists in estimating an agent's position exploiting knowledge about its motion model (typically its kinematics model where the velocity is a known input), a range measurement from a point and eventually other sensor readings related to the vehicle's attitude. The challenge of using single range information for localization is related to the fact that traditional trilateration algorithms used in systems as the Global Positioning System (GPS), long base line (LBL) or ultra short base line (USBL) underwater navigation systems are ill posed when only range from a single point should be known. Yet indeed fusing information from a motion model of the agent (including velocity and attitude) and a single range measurement can be sufficient to estimate the position of the agent. Finding the conditions on the agent's motion state that allow to estimate its position from a single range measurement is an observability problem that needs to be tackled in order to eventually design an observer. Given that range is a nonlinear function of the position, even if the motion model of the vehicle should be linear, the observability issue is inherently nonlinear. A major contribution on observability for nonlinear systems is [6] where the fundamental ideas and results about local and weakly local observability are described: single range localization studies building on differential geometric tools need to tackle the difficulties related to local and weakly local observability as opposed to the global observability concept known for linear systems. Such issues are clearly addressed, by example, in references [7], [8] and [9].

Single range aided localization is particularly relevant in cooperative navigation applications where a team of vehicles

This work was partially supported by MIUR (Italian Ministry of Education, Universities and Research) under the PRIN project MARIS: Marine Autonomous Robotics for InterventionS, call of year 2010-2011, prot. 2010FBLHRJ.

The authors are with Dipartimento Ingegneria Innovazione - ISME node - Università del Salento, via Monteroni, 73100 Lecce - Italy name. lastnamedunisalento.it needs to perform collaborative motion control tasks while possibly performing relative localization exploiting intravehicle communication to measure relative ranges. Such problems arise in several underwater robotics scenarios [10] [11] [12] as well as in more general settings [13]. Moreover, the problem of range based localization is technically similar to the problem of source localization where a vehicle knowing its own position is asked to estimate the position of a source (or target) from which it acquires range measurements [14] [15]. The localization solution described in this paper was partially inspired by the work in [15].

The single range navigation problem is of particular interest for underwater navigation applications where range measurements are typically acquired through acoustic time of flight based sensors: the data so collected is often corrupted by outliers. Indeed the issue of designing outlier robust underwater navigation systems is of great importance and it has been addressed, for example, in [16] [17] [18] [19]. The single range navigation problem here addressed is solved using a novel outlier robust state estimation filter building on an entropy based residual loss function [20]. The resulting state estimator has a predictor / corrector structure and its performance is compared to a standard Kalman filter in simulation.

The paper is organized as follows: a brief state of the art analysis relative to the single range observability problem is addressed in section II. Then the main ideas and methods used to solve the single range observability problem are described in section III. A Kalman filter solution for estimating the state is described in section IV where numerical simulation results are also reported. The novel outlier robust state estimator is described in section $\mathrm{V}$ that includes some numerical simulation examples. Finally, conclusions are summarized in section VI.

\section{Alternative APPROACHES FOR SINGLE RANGE OBSERVABILITY ANALYSIS}

A milestone contribution in the area of single range localization is given by the work of Batista et al. [21] [22]: they propose to study the single range localization problem of an agent subject to a constant, but unknown, drift velocity through an augmented state approach. The original nonlinear system (where the state belongs to $\mathbb{R}^{6}$ and is made of the agent's position and unknown drift velocity components) is transformed in a linear time varying (LTV) system in $\mathbb{R}^{9}$ through an augmented state technique. This leads to the remarkable result of allowing to study the global observability properties of the system with well known Gramian based tools of LTV systems theory [23] and 
of designing a Kalman filter for global state estimation. The LTV system derived in [21] [22] is of the form

$$
\begin{aligned}
& \dot{\mathbf{z}}=A(t, \mathbf{u}(t), y(t)) \mathbf{z}+B \mathbf{u}(t) \\
& y(t)=C \mathbf{z}
\end{aligned}
$$

namely, the system matrix $A(t)$ explicitly depends, among the rest, on the output $y(t)$ that is the range measurement. More precisely, the $A(t)$ matrix is a function of terms proportional to $1 / y(t)$ : this poses both fundamental as well as implementation issues. From a theoretical perspective, assuming that the output $y(t)$ should be affected by additive noise, the dependency of $A(t)$ from $y(t)$ implies that some of its entries are stochastic and that the model uncertainty on the state equation (1) could not be possibly assumed to be only additive as is usually done within the theory of Kalman filtering. As a consequence assuming additive gaussian noise on the state and output equations (1) - (2), the associated Kalman state estimator is not guaranteed to be optimal in the usual sense. Indeed the numerical examples provided in [22] confirm that the Kalman filter estimates converge to the true state variables, but there is no a priori guarantee that the estimate is optimal in terms of estimate covariance. A second potential difficulty arising from the structure of equations (1) - (2) is related to the eventual stability analysis of the Kalman filter on equations (1) - (2) coupled with a motion controller. Indeed the presence of the output $y(t)$ in the matrix $A(t)$ does not allow to exploit in a straightforward fashion the standard separation principle used within linear systems theory to study the convergence and stability of state estimation filters coupled with feedback controllers.

As for the implementation of the Kalman filter described in [22], given the dependency of some entries of $A(t)$ from $1 / y(t)$, it is necessary to assume that $y(t) \neq 0$ at all times. Indeed in [22] it is assumed that $y(t)$ has strictly positive, finite, lower and upper bounds: yet in real application scenarios with unpredictable sensor noise and outliers one would need to pre-filter the output in order to guarantee the absence of numerical issue related to exceedingly small or null $y$ readings.

In the light of the above observations and inspired by the work in [15] and [22], this paper describes an alternative approach to address the single range localization problem. As a result, the same problem addressed in [22] is globally solved by introducing an LTV system of the form

$$
\begin{aligned}
& \dot{\mathbf{z}}=A \mathbf{z}+B \mathbf{u}(t) \\
& y(t)=C(\mathbf{u}(t)) \mathbf{z}
\end{aligned}
$$

namely where the state equation is completely linear time invariant (LTI) and has dimension 8 rather than 9 . The output equation is still LTV, but has a very simple structure. The proposed method does not build on state augmentation techniques, but rather exploits the structure of the original state equations expressed in an inertial frame as opposed to the body frame formulation used in [22]. Within the proposed solution, given the LTI nature of the state equation (3), the difficulties related to the dependency of $A(t)$ in equation
(1) from $1 / y(t)$ are completely removed. Also notice that the output matrix $C(t)$ in equation (4) within the solution presented in this paper depends on the input $\mathbf{u}(t)$, but not on the output $y(t)$ : as a consequence an additive measurement noise would not affect the entries of $C(t)$ nor of $A$ and $B$ hence preserving the optimality of a Kalman filter as a state estimator as long as noise is gaussian (and the input $\mathbf{u}(t)$ is perfectly known, i.e. noise free). Indeed, as in [22], the localization problem can be globally solved with a standard Kalman filter with dimension 8 instead of 9 . Moreover, given the extremely simple structure of equations (3) - (4), the observability analysis is extremely simple and it allows to derive necessary and sufficient observability conditions on the agent input (i.e. its velocity).

\section{Single RANGE LOCALIZATION IN THE PRESENCE OF CONSTANT AND UNKNOWN CURRENTS}

Consider an agent (or a vehicle, in the following) with position given by vector $\mathbf{x}$ and a source (or navigation reference) with fixed inertial position s. Denoting with

$$
\mathbf{r}:=\mathbf{s}-\mathbf{x}
$$

the relative position of the source with respect to the agent it is assumed that this can access the measurement $y$ given by squared norm of $\mathbf{r}$, namely

$$
y=\|\mathbf{r}\|^{2} .
$$

Moreover, denoting with $\{I\}$ and $\{B\}$ an earth fixed and body fixed frames respectively, it is assumed that the agent has access to a measurement of its attitude, namely it can measure the rotation matrix ${ }^{I} R_{B} \in \mathrm{SO}(3)$ thanks to an on board navigation system (Attitude and Heading Reference System - AHRS). The agent velocity is given by a superposition of a drift term $\mathbf{v}_{f}$ and a controlled (input) term $\mathbf{v}_{r}$ that model a constant ocean current and a commanded velocity with respect to the water respectively. In practice the velocity term $\mathbf{v}_{r}$ for underwater vehicles can be measured by an on board navigation sensor as a Doppler Velocity Logger (DVL). The resulting agent motion model expressed in the $\{I\}$ frame is thus

$$
\begin{aligned}
\dot{\mathbf{x}} & =\mathbf{v}_{r}+\mathbf{v}_{f} \\
\dot{\mathbf{v}}_{f} & =\mathbf{0} \\
\dot{\mathbf{s}} & =\mathbf{0} .
\end{aligned}
$$

Consequently, the agents state equations in terms of the relative position $\mathbf{r}$ expressed in the fixed frame $\{I\}$ result in:

$$
\begin{aligned}
\dot{\mathbf{r}} & =-\mathbf{v}_{f}-\mathbf{v}_{r} \\
\dot{\mathbf{v}}_{f} & =\mathbf{0} \\
y & =\|\mathbf{r}\|^{2} .
\end{aligned}
$$

The problem addressed in the paper can thus be formulated as follows 


\section{Problem formulation}

Given the linear state equations $(10$ - 11) for the state vector $\left(\mathbf{r}^{\top}, \mathbf{v}_{f}^{\top}\right)^{\top}$ and the nonlinear scalar output $y$ in equation (12) determine the conditions on the input $\mathbf{v}_{r}$ that guarantee observability of the state and design a state estimator for $\left(\mathbf{r}^{\top}, \mathbf{v}_{f}^{\top}\right)^{\top}$ that is robust to possible outliers in the output $y$.

This problem corresponds to the one addressed in [22] with the only difference that here it is formulated in the inertial frame $\{I\}$ rather than in the body fixed frame $\{B\}$. Yet it should be noticed that the available information in the two set-ups is identical as both formulations require to have access to the rotation matrix ${ }^{I} R_{B} \in \mathrm{SO}(3)$. In particular, in the present paper ${ }^{I} R_{B}$ is needed to recover $\mathbf{v}_{r}$ in the $\{I\}$ frame from its measurement in the $\{B\}$ frame through a DVL (or other on board navigation sensors as GPS).

To tackle the formulated observability problem consider the integral of equation (10)

$$
\begin{aligned}
\mathbf{r}(t)-\mathbf{r}_{0} & =-\mathbf{v}_{f} t-\int_{0}^{t} \mathbf{v}_{r}(\tau) d \tau= \\
& =-\mathbf{v}_{f} t-\boldsymbol{I}_{\mathbf{v}_{r}}(t)
\end{aligned}
$$

having defined $\boldsymbol{I}_{\mathbf{v}_{r}}(t) \in \mathbb{R}^{3 \times 1}$ as

$$
\boldsymbol{I}_{\mathbf{v}_{r}}(t):=\int_{0}^{t} \mathbf{v}_{r}(\tau) d \tau
$$

and

$$
\mathbf{r}_{0}:=\left.\mathbf{r}(t)\right|_{t=0} .
$$

Equation (13) allows to compute

$\left(\mathbf{r}(t)+\boldsymbol{I}_{\mathbf{v}_{r}}(t)\right)^{\top}\left(\mathbf{r}(t)+\boldsymbol{I}_{\mathbf{v}_{r}}(t)\right)=\left(\mathbf{r}_{0}-\mathbf{v}_{f} t\right)^{\top}\left(\mathbf{r}_{0}-\mathbf{v}_{f} t\right)$

implying

$$
\begin{array}{r}
\|\mathbf{r}(t)\|^{2}+\left\|\boldsymbol{I}_{\mathbf{v}_{r}}(t)\right\|^{2}+2 \boldsymbol{I}_{\mathbf{v}_{r}}^{\top}(t) \mathbf{r}(t)= \\
=\left\|\mathbf{r}_{0}\right\|^{2}+\left\|\mathbf{v}_{f}\right\|^{2} t^{2}-2\left(\mathbf{r}_{0}^{\top} \mathbf{v}_{f}\right) t
\end{array}
$$

namely

$$
\begin{array}{r}
\|\mathbf{r}(t)\|^{2}-\left\|\mathbf{r}_{0}\right\|^{2}+\left\|\boldsymbol{I}_{\mathbf{v}_{r}}(t)\right\|^{2}= \\
=-2 \boldsymbol{I}_{\mathbf{v}_{r}}^{\top}(t) \mathbf{r}(t)-2\left(\mathbf{r}_{0}^{\top} \mathbf{v}_{f}\right) t+\left\|\mathbf{v}_{f}\right\|^{2} t^{2} .
\end{array}
$$

Notice that the left hand side of equation (16) is made of all known terms and it can be used as a new output map

$$
\begin{aligned}
\bar{y}(t) & =\|\mathbf{r}(t)\|^{2}-\left\|\mathbf{r}_{0}\right\|^{2}+\left\|\boldsymbol{I}_{\mathbf{v}_{r}}(t)\right\|^{2} \\
& =y(t)-y_{0}+\left\|\boldsymbol{I}_{\mathbf{v}_{r}}(t)\right\|^{2}
\end{aligned}
$$

and the right hand side of equation (16) can be expressed as a linear time varying (LTV) term in the new state variable $\mathbf{z} \in \mathbb{R}^{8 \times 1}$

$$
\mathbf{z}=\left(\mathbf{r}^{\top},\left(\mathbf{r}_{0}^{\top} \mathbf{v}_{f}\right),\left\|\mathbf{v}_{f}\right\|^{2}, \mathbf{v}_{f}^{\top}\right)^{\top}
$$

i.e.

$$
\begin{aligned}
\bar{y}(t) & =C(t) \mathbf{z}= \\
& =\left[\begin{array}{llll}
-2 \boldsymbol{I}_{\mathbf{v}_{r}}^{\top}(t) & -2 t & t^{2} & 0 \\
1 \times 3
\end{array}\right] \mathbf{z} .
\end{aligned}
$$

Given the definition of $\mathbf{z}$ in equation (18) and the model (10) - (11), its dynamic equation is linear time invariant (LTI):

$$
\dot{\mathbf{z}}=A \mathbf{z}+B \mathbf{v}_{r}
$$

namely

$$
\begin{aligned}
\dot{\mathbf{z}} & =\frac{d}{d t}\left(\begin{array}{c}
\mathbf{r} \\
\left(\mathbf{r}_{0}^{\top} \mathbf{v}_{f}\right) \\
\left\|\mathbf{v}_{f}\right\|^{2} \\
\mathbf{v}_{f}
\end{array}\right)= \\
= & {\left[\begin{array}{cccc}
0_{3 \times 3} & 0_{3 \times 1} & 0_{3 \times 1} & -I_{3 \times 3} \\
0_{1 \times 3} & 0 & 0 & 0_{1 \times 3} \\
0_{1 \times 3} & 0 & 0 & 0_{1 \times 3} \\
0_{3 \times 3} & 0_{3 \times 1} & 0_{3 \times 1} & 0_{3 \times 3}
\end{array}\right]\left(\begin{array}{c}
\mathbf{r} \\
\left(\mathbf{r}_{0}^{\top} \mathbf{v}_{f}\right) \\
\left\|\mathbf{v}_{f}\right\|^{2} \\
\mathbf{v}_{f}
\end{array}\right)+} \\
+ & {\left[\begin{array}{c}
-I_{3 \times 3} \\
0_{1 \times 3} \\
0_{1 \times 3} \\
0_{3 \times 3}
\end{array}\right] \mathbf{v}_{r} . }
\end{aligned}
$$

The range-only localization problem of estimating $\mathbf{r}$ and the current velocity $\mathbf{v}_{f}$ from a measurement of $\|\mathbf{r}\|^{2}$ in equations (10) - (12) is hence reduced to a state estimation problem on a linear time invariant state equation (20) - (21) with an LTV output map (19), namely

$$
\left\{\begin{array}{l}
\dot{\mathbf{z}}=A \mathbf{z}+B \mathbf{v}_{r} \\
\bar{y}(t)=C(t) \mathbf{z}
\end{array}\right.
$$

The LTI state equations, moreover, have a very simple structure. As anticipated in section I, notice that this results is similar to the one described in [22], but with a few significant differences: the state matrix $A$ does not depend on the output and is actually LTI rather than LTV. Only the output map is time varying and depends on the vehicle's velocity. Moreover the state vector has dimension 8 rather than 9 . As a consequence, the Gramian observability matrix to be used for observability analysis has a simpler structure as well as the resulting observer that can be chosen to have a Kalman filter structure.

Estimating $\mathbf{z}$ will result in estimating both $\mathbf{r}$ and the current velocity $\mathbf{v}_{f}$. Moreover, in case that the absolute position $\mathbf{s}$ of the source should be known a priori, by estimating $\mathbf{r}$ the absolute position of the vehicle could also be computed as $\mathbf{x}=\mathbf{s}-\mathbf{r}$.

\section{A. Observability analysis in the presence of currents}

The observability properties of system (22) can be studied through the observability Gramian

$$
G(t)=\int_{0}^{t} e^{A^{\top} \tau} C^{\top}(\tau) C(\tau) e^{A \tau} d \tau
$$

Given the structure of the $A$ matrix in equation (21), notice that $A^{2}=0_{8 \times 8}$ implying that the exponential matrix $\exp (A t)$ is simply

$$
e^{A t}=I_{8 \times 8}+A t
$$

such that $C(t) \exp (A t)$ results in

$$
C(t) e^{A t}=\left[\begin{array}{llll}
-2 \boldsymbol{I}_{\mathbf{v}_{r}}^{\top}(t) & -2 t & t^{2} & 2 t \boldsymbol{I}_{\mathbf{v}_{r}}^{\top}(t)
\end{array}\right]
$$


and

$$
\begin{gathered}
\exp \left(A^{\top} \tau\right) C^{\top}(\tau) C(\tau) \exp (A \tau)= \\
=\left[\begin{array}{cccc}
4 \boldsymbol{I}_{\mathbf{v}_{r} r} \boldsymbol{I}_{\mathbf{v} r}^{\top} & 4 \tau \boldsymbol{I}_{\mathbf{v}_{r}} & -2 \tau^{2} \boldsymbol{I}_{\mathbf{v}_{r}} & -4 \tau \boldsymbol{I}_{\mathbf{v}_{r}} \boldsymbol{I}_{\mathbf{v}_{r}}^{\top} \\
4 \tau \boldsymbol{I}_{\mathbf{v}_{r}}^{\top} & 4 \tau^{2} & -2 \tau^{3} & -4 \tau^{2} \boldsymbol{I}_{\mathbf{v}_{r}}^{\top} \\
-2 \tau^{2} \boldsymbol{I}_{\mathbf{v}_{r}}^{\top} & -2 \tau^{3} & \tau^{4} & 2 \tau^{3} \boldsymbol{I}_{\mathbf{v}_{r}}^{\top} \\
-4 \tau \boldsymbol{I}_{\mathbf{v}_{r}} \boldsymbol{I}_{\mathbf{v}_{r}}^{\top} & -4 \tau^{2} \boldsymbol{I}_{\mathbf{v}_{r}} & 2 \tau^{3} \boldsymbol{I}_{\mathbf{v}_{r}} & 4 \tau^{2} \boldsymbol{I}_{\mathbf{v}_{r}} \boldsymbol{I}_{\mathbf{v}_{r}}^{\top}
\end{array}\right]
\end{gathered}
$$

where the dependency of $\boldsymbol{I}_{\mathbf{v}_{r}}$ from $t$ has been omitted for the sake of notation compactness.

As for the observability conditions, following standard results for LTV systems [23], the model in equation (22) will be completely observable in the time interval $[0, t]$ if and only if the Gramian given by equations (23) and (26) has full rank. Moreover, the structure of equation (26) implies that a necessary condition for the complete observability of (22) in the time interval $[0, t]$ is that

$$
G_{11}(t):=4 \int_{0}^{t} \boldsymbol{I}_{\mathbf{v}_{r}}(\tau) \boldsymbol{I}_{\mathbf{v}_{r}}^{\top}(\tau) d \tau
$$

has full rank, i.e. three. Overall, the observability properties in the presence of constant currents can be summarized as follows.

Main Result - Observability conditions for the continuous time case with constant current.

The model in equations (19) - (21) is observable on $[0, t]$ if and only if the velocity signal $\mathbf{v}_{r}$ guarantees that the Gramian in equations (23) and (26) has full rank. Moreover a necessary condition for full observability on $[0, t]$ is that the matrix $G_{11}(t) \in \mathbb{R}^{3 \times 3}$ in equation (27) has rank 3 .

\section{Proof of the Main Result}

The necessary and sufficient conditions on the Gramian in equations (23) and (26) follow from standard LTV systems theory [23]. As for the necessary condition on the rank of the matrix $G_{11}(t) \in \mathbb{R}^{3 \times 3}$ in equation (27) it results that if $G_{11}(t)$ should not be full rank on $[0, t]$, there would exist a constant vector $\boldsymbol{\nu} \in \mathbb{R}^{3 \times 1}, \boldsymbol{\nu} \neq \mathbf{0}$ such that $\boldsymbol{I}_{\mathbf{v}_{r}}(\tau)^{\top} \boldsymbol{\nu}=0 \quad \forall \quad \tau \in[0, t]$ : this implies that any vector parallel to $\mathbf{z}^{*}=\left(\alpha \boldsymbol{\nu}^{\top}, 0,0, \beta \boldsymbol{\nu}^{\top}\right)^{\top} \in \mathbb{R}^{8 \times 1}$ for any constant $\alpha, \beta \in \mathbb{R}$ would belong to the kernel of the Gramian (23) - (26) that, hence, would not be full rank. This proves that $\operatorname{rank}\left(G_{11}(t)\right)=3: G_{11}(t) \in \mathbb{R}^{3 \times 3}$ is defined in equation (27) is a necessary condition for the observability in $[0, t]$ of the model in equations (19) - (21).

Notice that the Main Results allow to consider optimal design issues of the vehicle's input $\mathbf{v}_{r}$ : in Kalman filtering theory, in fact, the observability Gramian is related to the estimate covariance and to the Fisher information matrix. Building on the Main Result, one could formulate optimal design problems for the input $\mathbf{v}_{r}$ aiming at maximizing, by example, metrics as the norm or the determinant, or the condition number of the resulting Fisher information matrix.

\section{KALMAN FILTER DESIGN}

With reference to the model in equation (22) assume that it is discretized and affected by state and output zero mean mutually independent disturbances respectively $\boldsymbol{\omega}_{k}$ and $\varepsilon_{k}$ with covariances

$$
\begin{aligned}
\operatorname{cov}\left(\boldsymbol{\omega}_{k}\right) & =E\left[\boldsymbol{\omega}_{k} \boldsymbol{\omega}_{k}^{\top}\right]=Q_{k} \\
\operatorname{cov}\left(\varepsilon_{k}\right) & =E\left[\varepsilon_{k}^{2}\right]=R_{k} .
\end{aligned}
$$

Denoting with $\hat{\mathbf{x}}_{k \mid k}$ the Kalman estimate at step $k$ and with $\hat{\mathbf{x}}_{k+1 \mid k}$ the model prediction, the localization Kalman filter can be designed.
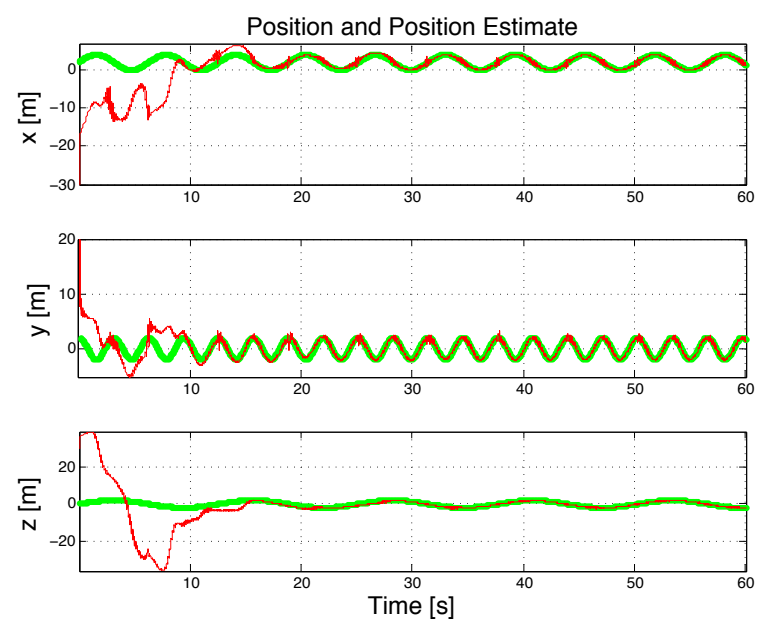

Fig. 1. Kalman filter estimation including currents: real (in green) and estimated (in red) components of $\mathbf{x}$.
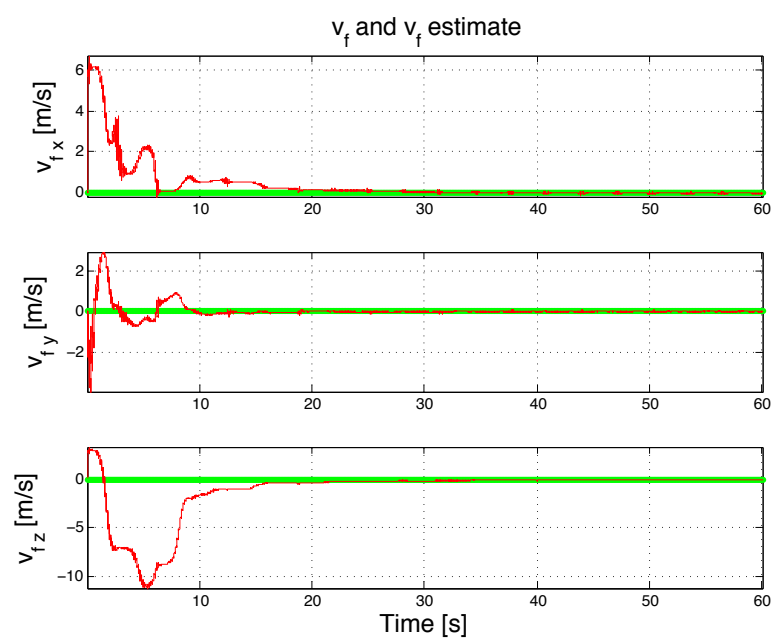

Fig. 2. Kalman filter estimation including currents: real (in green) and estimated (in red) components of the current velocity $\mathbf{v}_{f}$.

In particular, a numerical experiment is performed using the same agent velocity profile $\mathbf{v}_{r}$ used in the examples presented in [14] and [22] namely $\mathbf{v}_{r}=$ $(2 \cos (t),-4 \sin (2 t), \cos (t / 2))^{\top}(\mathrm{m} / \mathrm{s})$. The target $\mathbf{s}$ is $\mathbf{s}=$ 
$(2,3,1)^{\top}(\mathrm{m})$, the current is assumed null, $\mathbf{v}_{f}=\mathbf{0}(\mathrm{m} / \mathrm{s})$, and the initial position of the agent is $\mathbf{x}_{0}=(2,2,0)^{\top}$ (m) such that the inertial position of the agent by $\mathbf{x}(t)=(2+$ $2 \sin (t), 2 \cos (2 t), 2 \sin (0.5 t))^{\top}(\mathrm{m})$. Notice that, by direct calculation, the above $\mathbf{v}_{r}$ input satisfies the observability condition given in the Main Result. The covariances on the state $\mathbf{z}$ and output $\bar{y}(t)$ employed in the Kalman filter are $Q=$ $(1 e-2) \operatorname{diag}([1,1,1,1 e-4,1 e-6,(1 e-2),(1 e-2),(1 e-2))$ and $R=1$ respectively with proper units (i.e. $\left[\mathrm{m}^{2}\right]$ for position variables and $\left[(\mathrm{m} / \mathrm{s})^{2}\right]$ for velocity variables). The filter is initialized with a position $\hat{\mathbf{x}}_{0}=(-30,20,30)^{\top}[\mathrm{m}]$ as opposed to the real initial position $\mathbf{x}_{0}=(2,2,0)^{\top}[\mathrm{m}]$ and a current estimate $\hat{\mathbf{v}}_{f}=(0.1,-0.1,0.1)^{\top}[\mathrm{m} / \mathrm{s}]$ as opposed to the real null current. Denoting with $T_{s}$ the sampling time (that was $(1 / 750)$ [s] in the described example), the Kalman filter equations result in:

$$
\begin{aligned}
& A_{d}=\left(I_{8 \times 8}+T_{s} A\right) \\
& B_{d}=T_{s} B \\
& \hat{\mathbf{z}}_{k+1 \mid k}=A_{d} \hat{\mathbf{z}}_{k \mid k}+B_{d} \mathbf{v}_{r k} \\
& P_{k+1 \mid k}=A_{d} P_{k \mid k} A_{d}^{\top}+Q_{k} \\
& K=\left(P_{k+1 \mid k}^{-1}+C_{k+1}^{\top} R_{k+1}^{-1} C_{k+1}\right)^{-1} C_{k+1}^{\top} R_{k+1}^{-1} \\
& \hat{\mathbf{z}}_{k+1 \mid k+1}=\hat{\mathbf{z}}_{k+1 \mid k}+K\left(\bar{y}_{k+1}-C_{k+1} \hat{\mathbf{z}}_{k+1 \mid k}\right)
\end{aligned}
$$

where $Q_{k}$ and $R_{k}$ were constant and equal to the values reported above.

The resulting time evolution of the agent position $\mathbf{x}=\mathbf{s}-\mathbf{r}$ and its estimate $\hat{\mathbf{x}}=\mathbf{s}-\hat{\mathbf{r}}$ are plotted in figure 1 while the current estimate is plotted in figure 2.

\section{A. Discussion}

As already noticed, the proposed solution allows to design a Kalman filter for state estimation on a system where all the system matrices $(A, B$ and $C(t))$ are not affected by measurement noise as they do not depend from the output. This preserves the optimality of the Kalman filter as a state estimator in case of additive gaussian noise on the output and state equations. Yet the new output $\bar{y}(t)$ in equation (17) depends on the very first measurement $y(0)$. This dependency can impact on the robustness of the solution as a single bad measurement (as an outlier) at $t=0$ will affect the output for ever. A remedy to this issue can be found by periodically resetting the initial measurement $y(0)$ with $y(t)$. In the discrete time case this would correspond to periodically mapping $y_{0} \longrightarrow y_{k^{*}}$ as if the measurement had started at step $k^{*}$ while the state estimate $\hat{\mathbf{x}}_{k \mid k}$ continues its update dynamics. A detailed analysis of this implementation detail goes beyond the scope of this paper and will not be addressed further, but it will be subject to future investigation. Besides this issue, notice that outliers are likely to be present in the range measurements in particular when acoustic sensors are employed. As a consequence the use of an outlier robust estimator rather than a standard Kalman filter is eventually better suited for the current application.

\section{OUtLIER Robust STATE ESTIMATION}

Whatever the application of the single range localization is (land, aerial or marine), the range measurements are often contaminated by outliers, namely data points that cannot be modeled by a single (eventually Gaussian) probability distribution function. If the noise were purely Gaussian, a Kalman filter would provide the optimal solution. Unfortunately, most often this is not the case and robustness to outliers is a key issue. To address this, one class of approaches exploits the equivalence between the Kalman filter and a weighted least square regression problem. It is known that the Kalman filter can be derived as a solution to the following minimization problem:

$$
\begin{aligned}
\hat{\mathbf{z}}_{k+1 \mid k+1}=\arg \min \\
\\
\quad \frac{1}{\mathbf{z}_{k+1}}\left(\mathbf{z}_{k+1}-\hat{\mathbf{z}}_{k+1 \mid k}\right)^{T} P_{k+1 \mid k}^{-1}\left(\mathbf{z}_{k+1}-\hat{\mathbf{z}}_{k+1 \mid k}\right)+ \\
+\frac{1}{2}\left(\bar{y}_{k+1}-C_{k+1} \mathbf{z}_{k+1}\right)^{T} R_{k+1}^{-1}\left(\bar{y}_{k+1}-C_{k+1} \mathbf{z}_{k+1}\right)
\end{aligned}
$$

where $R_{k+1}$ is the covariance associated to the measurement $\bar{y}_{k+1}$ and $P_{k+1 \mid k}$ is the covariance of the (model) predicted state $\hat{\mathbf{z}}_{k+1 \mid k}$. In [24], [25] and [26] the authors solve this minimization problem in a robust manner replacing the second term of the objective function by robustifying functions used in the methodology of M-estimation (e.g., the Huber function [27]). They express the solution as a weighted least square approximation, where each weight indicates its contribution to the state estimate. The robustness is achieved trying to give a finite weight to single residuals that exceed a threshold. Each residual contributes to the objective function based on its only value regardless the overall distribution. Other approaches as [28] and [29] model the observation noise through a heavy-tailed distribution assigning outliers a non-negligible probability: interestingly the resulting estimators still result in a weighted [28] or iterative re-weighted [29] least squares solution. A robust alternative technique is here proposed based on the robust parameter identification method known as LEL (Least Entropy-Like) [20]. The basic idea is to estimate the state minimizing the following non linear objective function

$$
\begin{aligned}
\hat{\mathbf{z}}_{k+1 \mid k+1}=\arg \min _{\mathbf{z}_{k+1}} J_{k+1} \\
J_{k+1}=\underbrace{\frac{1}{2}\left(\left(\mathbf{z}_{k+1}-\hat{\mathbf{z}}_{k+1 \mid k}\right)^{T} P_{k+1 \mid k}^{-1}\left(\mathbf{z}_{k+1}-\hat{\mathbf{z}}_{k+1 \mid k}\right)\right)}_{J_{\text {dynamical model }}}+ \\
+\underbrace{\alpha H_{k+1}\left(r_{1}, \ldots, r_{k+1}\right)}_{J_{L E L}}
\end{aligned}
$$

where $r_{i}=\bar{y}_{i}-C_{i} \hat{\mathbf{z}}_{i}, i=1, \ldots, k+1$ denotes the $\mathrm{i}$-th residual and $H_{k+1}(\cdot)$ represents an entropy based residual loss function. Define $D$ as the least squares cost

$$
D=\sum_{j=1}^{k+1} r_{j}^{2}
$$


and the relative squared residual $q_{i}$ as

$$
\text { if } D \neq 0 \Rightarrow q_{i}:=\frac{r_{i}{ }^{2}}{\sum_{j=1}^{k+1} r_{j}{ }^{2}}: q_{i} \in[0,1] \text { and } \sum_{i=1}^{k+1} q_{i}=1 \text {, }
$$

the residual loss function has the following form:

$$
H_{k+1}= \begin{cases}0 & \text { if } D=0 \\ -\frac{1}{\log (k+1)} \sum_{i=1}^{k+1} q_{i} \log q_{i} & \text { otherwise. }\end{cases}
$$

The main difference respect to the Kalman filter and the methods based on M-estimators relies on the structure of the second term of the objective function (37). The aim of such loss function is to give a 'global' measure of the scatter of the relative squared residuals. The idea behind the estimator is to make the relative squared residuals 'as little equally distributed as possible'. If this is the case, 'most' residuals are small (with respect to the normalization constant $\mathrm{D}$, i.e. the Least Squares cost) and 'a few' of the residuals are large. Data points corresponding to these large residual are outlier candidates. It is worth highlighting that the structure of the LEL entropy-based loss function can not be resembled to the methodology of M-estimators. Indeed according to the definition of M-estimators, the contribution to the objective function of the i-the residual does not depend on the other residuals. This is not the case of the proposed estimator, since all residuals contribute to the objective function of the i-the residual through the relative squared residual $q_{i}$.

It should be noticed that $H_{k+1}(\cdot) \in[0,1]$ by construction, so the parameter $\alpha$ in (37) is to be regarded as a tuning factor needed to make the two terms of $J_{k+1}$ comparable.

In order to find the solution of the minimization problem (37), $H_{k+1}(\cdot)$ can be approximated in a neighborhood of $\hat{\mathbf{z}}_{k}$ with a quadratic function by means of its second order Taylor series expansion. Thus, setting the gradient of such second order Taylor expansion with respect to $\mathbf{z}_{k+1}$ equal to zero, the filter equations result in:

$$
\begin{aligned}
& \hat{\mathbf{z}}_{k+1 \mid k}=A_{d} \hat{\mathbf{z}}_{k}+B_{d} \mathbf{v}_{r k} \\
& P_{k+1 \mid k}=A_{d} P_{k \mid k} A_{d}^{T}+Q_{k} \\
& K_{k+1}=\left(P_{k+1 \mid k}^{-1}+\alpha \mathcal{H}\left[H_{k+1}\left(\hat{\mathbf{z}}_{k}\right)\right]\right)^{-1} \alpha \mathcal{H}\left[H_{k+1}\left(\hat{\mathbf{z}}_{k}\right)\right] \\
& \hat{\mathbf{z}}_{k+1}=\hat{\mathbf{z}}_{k+1 \mid k}+K_{k+1}\left(\hat{\mathbf{z}}_{k}-\hat{\mathbf{z}}_{k+1 \mid k}\right)+ \\
& -\left(P_{k+1 \mid k}^{-1}+\alpha \mathcal{H}\left[H_{k+1}\left(\hat{\mathbf{z}}_{k}\right)\right]\right)^{-1} \alpha \nabla H_{k+1}\left(\hat{\mathbf{z}}_{k}\right) \\
& P_{k+1 \mid k+1}=\left(P_{k+1 \mid k}^{-1}+\alpha \mathcal{H}\left[H_{k+1}\left(\hat{\mathbf{z}}_{k}\right)\right]\right)^{-1}
\end{aligned}
$$

where the gradient and hessian $\mathcal{H}(\cdot)$ of the LEL cost function computed about the point $\hat{\mathbf{z}}_{k}$ have been denoted respectively as

$$
\begin{array}{r}
\left.\nabla_{\mathbf{z}_{k+1}} H_{k+1}(\cdot)\right|_{\mathbf{z}_{k+1}=\hat{\mathbf{z}}_{k}}=\nabla H_{k+1}\left(\hat{\mathbf{z}}_{k}\right) \\
\left.\mathcal{H}\left[H_{k+1}(\cdot)\right]\right|_{\mathbf{z}_{k+1}=\hat{\mathbf{z}}_{k}}=\mathcal{H}\left[H_{k+1}\left(\hat{\mathbf{z}}_{k}\right)\right]
\end{array}
$$

Notice that besides the term

$$
-\left(P_{k+1 \mid k}^{-1}+\alpha \mathcal{H}\left[H_{k+1}\left(\hat{\mathbf{z}}_{k}\right)\right]\right)^{-1} \alpha \nabla H_{k+1}\left(\hat{\mathbf{z}}_{k}\right)
$$

in equation (44), the resulting state estimator filter has a predictor - corrector structure as the standard Kalman filter. Indeed the term in equation (48) will be null if $\hat{\mathbf{z}}_{k}$ is a local minima of $H_{k+1}$. The prediction equations are responsible for projecting forward (in time) the current state and error covariance estimates to obtain the a priori estimates for the next time step, whereas the corrector equations are responsible for robust incorporating a new measurement into the a priori estimate to obtain an improved a posteriori estimate. Moreover, this method is characterized by a low computational effort making it suitable for real-time applications. Further details about the implementation and derivation of the filter are omitted due to the lack of space.

In order to illustrate the robustness properties of the algorithm, it has been tested on the same numerical experiment performed in the previous section with the addition of some outliers in the range measurements to make them more realistic. Indeed, in the case of acoustic sensors for range acquisitions, a significant source of non-Gaussian noise is multipath, therefore to obtain a realistic simulation the outliers have been generated reproducing this phenomena. Specifically, the outliers has been assigned as a multiplevalued of the real range according to the following measurement model:

$$
\begin{array}{r}
y_{i}(t)=\left(\mu_{i}\left\|r_{i}(t)\right\|\right)^{2}+\epsilon_{i} \\
\epsilon_{i} \sim N(0, R) \\
\mu_{i}= \begin{cases}1 & \text { for inliers } \\
2 & \text { for outliers. }\end{cases}
\end{array}
$$

In the numerical experiment each measure has been reproduced as outlier $\left(\mu_{i}=2\right)$ with probability $0.1 \%$. On top of such outliers, a set of 50 consecutive outliers has been reproduced at time 40 seconds in order to test the performance of the filter in extreme conditions. The resulting evolution of the agents position and its estimate through both filters are plotted in figure 3 together with the estimate of the current and the measured output. The plots in figure 3 show how sensitive the standard Kalman filter is to outliers as compared to the proposed LEL filter that exhibits a much better performance when outlying data occur (either isolated or consecutive). Notice that the Kalman filter generating the estimates in figure 3 is the ideal one, i.e. the measurement and state noise covariance matrices used to compute the Kalman gain are precisely the ones used in the model.

Besides the reported results, additional extensive simulations have been performed confirming that the LEL state estimation filter outperforms the Kalman estimator in terms of outlier robustness.

\section{CONCLUSIONS}

The problem of single range based localization for the kinematics model of a $3 D$ vehicle was addressed in this paper. The problem is relevant in several filed robotics applications, particularly in underwater scenarios where ranges are measured acoustically and alternative radio frequency based localization devices as GPS are not available. Single range based localization techniques allow to avoid using 

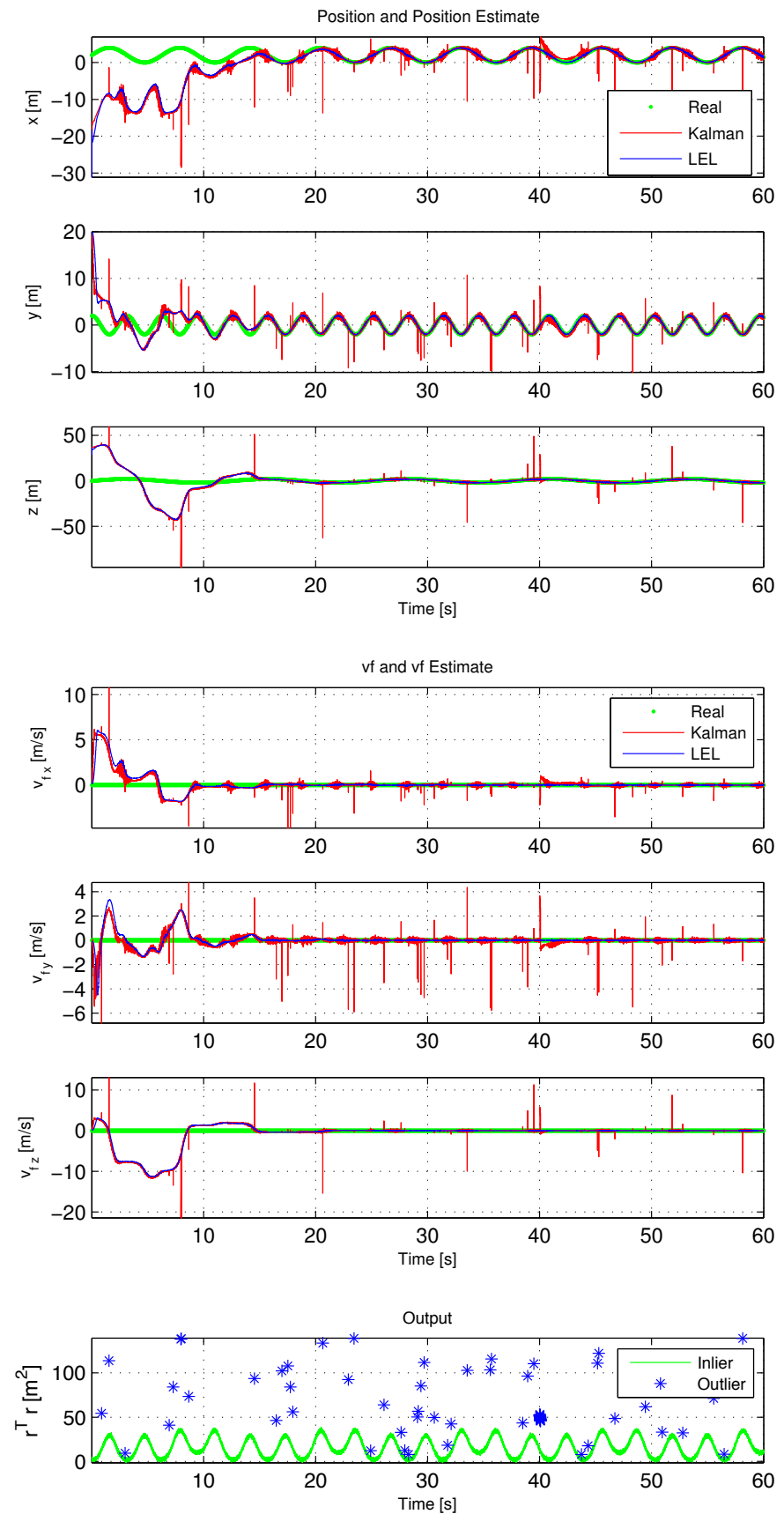

Fig. 3. Kalman and LEL filter estimations in presence of outliers: real and estimated components of $\mathbf{x}$ and $\mathbf{v}_{f}$; observed noisy output data with outliers.

trilateration based devices such as long base line (LBL) transponders that are very demanding in terms of cost and deployment effort. The vehicle is assumed to be equipped with standard on board navigation sensors as a doppler velocity logger DVL and an attitude heading reference system AHRS allowing to access the linear and angular vehicle velocities as well as the system's attitude, i.e. the rotation matrix ${ }^{I} R_{B}$ from body frame $\{B\}$ to the earth fixed frame $\{I\}$. The localization problem addressed is equivalent to the one presented in [22] and it explicitly accounts for the effects of a constant, but unknown, ocean current that is estimated together with the vehicle position. The proposed solution allows to address the observability analysis and the state estimation filter design on a linear time invariant state equation defined on $\mathbb{R}^{8}$ with a time varying scalar output equation. In this respect the proposed solution resembles the one in [22] where the original problem was transformed in a linear time varying state equation defined on $\mathbb{R}^{9}$ with a linear time invariant scalar output equation. Yet contrary to this previous solution, the state equation matrix $A$ does not depend on the inverse of the output $y(t)$ hence preserving the optimality of the Kalman filter in case of additive gaussian 
noise on the state and output equations. Moreover, the simple structure of the derived linear system for observability analysis allows to define straightforward necessary and sufficient observability conditions. To cope with possible outliers in the range measurements, a robust predictor - corrector state estimator has been proposed. Such filter builds on the novel Least Entropy-Like (LEL) parameter estimation paradigm illustrated in [20] that significantly departs from alternative robust state estimators based on M-estimators or heavy-tailed noise distributions.

The proposed solution can be applied in underwater cooperative navigation applications, sensor networks and source localization problems.

\section{REFERENCES}

[1] A. Martinelli and R. Siegwart, "Observability analysis for mobile robot localization," in Intelligent Robots and Systems, 2005. (IROS 2005). 2005 IEEE/RSJ International Conference on, aug. 2005, pp. 1471 - 1476. [Online]. Available: http://dx.doi.org/10.1109/IROS. 2005.1545153

[2] X. S. Zhou and S. I. Roumeliotis, "Robot-to-robot relative pose estimation from range measurements," IEEE Transactions on Robotics, vol. 24, no. 6, pp. 1379-1393, 2008. [Online]. Available: http://dx.doi.org/10.1109/TRO.2008.2006251

[3] Z. M. Kassas and T. E. Humphreys, "Observability analysis of opportunistic navigation with pseudorange measurements," in AIAA Guidance, Navigation, and Control Conference, AIAA GNC, 2012. [Online]. Available: http://dx.doi.org/10.2514/6.2012-4760

[4] T. Maki, T. Matsuda, T. Sakamaki, T. Ura, and J. Kojima, "Navigation method for underwater vehicles based on mutual acoustical positioning with a single seafloor station," IEEE Journal Of Oceanic Engineering, vol. 38, no. 1, pp. 167 - 177, 2013. [Online]. Available: http://dx.doi.org/10.1109/JOE.2012.2210799

[5] F. Arrichiello, G. Antonelli, A. P. Aguiar, and A. Pascoal, "Observabiliy metrics for the relative localization of AUVs based on range and depth measurements: theory and experiments," in Proceedings of the 2011 IEEE/RSJ International Conference on Intelligent Robots and Systems, IEEE-IROS 2011, Hilton San Francisco Union Square, San Francisco, CA, USA, September 2011. [Online]. Available: http://dx.doi.org/10.1109/IROS.2011.6094466

[6] R. Hermann and A. J. Krener, "Nonlinear controllability and observability," IEEE Transactions on Automatic Control, vol. 22, no. 5, pp. 728 - 740, October 1977. [Online]. Available: http://dx.doi.org/10.1109/TAC.1977.1101601

[7] J. Jouffroy and J. Reger, "An algebraic perspective to singletransponder underwater navigation," in Computer Aided Control System Design, 2006 IEEE International Conference on Control Applications, 2006 IEEE International Symposium on Intelligent Control. IEEE, 2006, pp. 1789-1794. [Online]. Available: http: //dx.doi.org/10.1109/CACSD-CCA-ISIC.2006.4776912

[8] A. Ross and J. Jouffroy, "Remarks on the observability of single beacon underwater navigation," in Int. Symp. on Unmanned Untethered Submersible Technology (UUST 05), Durham, NH, August 2005.

[9] A. S. Gadre and D. J. Stilwell, "Toward underwater navigation based on range measurements from a single location," in Proceedings of IEEE International Conference on Robotics and Automation, 2004 (ICRA 2004), New Orleans, LA, USA, 26 April - 1 May 20042004. [Online]. Available: http://dx.doi.org/10.1109/ROBOT.2004.1302422

[10] M. F. Fallon, G. Papadopoulos, J. J. Leonard, and N. M. Patrikalakis, "Cooperative AUV navigation using a single maneuvering surface craft,” I. J. Robotic Res., vol. 29, no. 12, pp. 1461-1474, 2010. [Online]. Available: http://dx.doi.org/10.1177/0278364910380760

[11] S. E. Webster, J. M. Walls, L. L. Whitcomb, and R. M. Eustice, "Decentralized extended information filter for single-beacon cooperative acoustic navigation: Theory and experiments," IEEE Transactions on Robotics, no. 99, pp. 1-18, 2013. [Online]. Available: http://dx.doi.org/10.1109/TRO.2013.2252857

[12] J. M. Soares, A. P. Aguiar, A. M. Pascoal, and A. Martinoli, "Joint ASV/AUV range-based formation control: Theory and experimental results," in 2013 IEEE International Conference on Robotics and Automation ICRA 2013, Karlsruhe, Germany, 6-10 May 2013.
[13] M. Cao, C. Yu, and B. D. Anderson, "Formation control using range-only measurements," Automatica, vol. 47, pp. 776-781, 2011. [Online]. Available: http://dx.doi.org/10.1016/j.automatica.2011.01. 067

[14] S. H. Dandach, B. Fidan, S. Dasgupta, and B. D. Anderson, "A continuous time linear adaptive source localization algorithm, robust to persistent drift," Systems \& Control Letters, vol. 59, pp. 7-16, 2009. [Online]. Available: http://dx.doi.org/10.1016/j.sysconle.2008.07.008

[15] G. Indiveri, P. Pedone, and M. Cuccovillo, "Fixed target 3D localization based on range data only: A recursive least squares approach," in Proceedings of the 2012 IFAC Workshop on Navigation, Guidance and Control of Underwater Vehicles, IFAC-NGCUV2012, Porto, Portugal, 10 - 12 April 2012, pp. 140 - 145. [Online]. Available: http://dx.doi.org/10.3182/20120410-3-PT-4028.00024

[16] J. Vaganay, J. Leonard, and J. Bellingham, "Outlier rejection for autonomous acoustic navigation," in Proceedings of the 1996 IEEE International Conference on Robotics and Automation, ICRA 1996, vol. 3. IEEE, 1996, pp. 2174-2181. [Online]. Available: http://dx.doi.org/10.1109/ROBOT.1996.506191

[17] S. Vike and J. Jouffroy, "Diffusion-based outlier rejection for underwater navigation," in Proceedings of MTS/IEEE OCEANS, 2005, vol. 2. IEEE, 2005, pp. 1037-1042. [Online]. Available: http://dx.doi.org/10.1109/OCEANS.2005.1639890

[18] A. Alcocer, P. Oliveira, and A. Pascoal, "Study and implementation of an ekf gib-based underwater positioning system," Control engineering practice, vol. 15, no. 6, pp. 689-701, 2007. [Online]. Available: http://dx.doi.org/10.1016/j.conengprac.2006.04.001

[19] J. Jouffroy and J. Opderbecke, "Underwater vehicle navigation using diffusion-based trajectory observers," Oceanic Engineering, IEEE Journal of, vol. 32, no. 2, pp. 313-326, 2007. [Online]. Available: http://dx.doi.org/10.1109/JOE.2006.880392

[20] G. Indiveri, "An entropy-like estimator for robust parameter identification," Entropy, vol. 11, no. 4, pp. 560-585, 2009. [Online]. Available: http://dx.doi.org/10.3390/e11040560

[21] P. Batista, C. Silvestre, and P. Oliveira, "Single beacon navigation: Observability analysis and filter design," in Proceedings of the 2010 American Control Conference, ACC2010. Marriott Waterfront, Baltimore, MD, USA: IEEE, June 30 - July 02 2010, pp. 6191-6196.

[22] - "Single range aided navigation and source localization: Observability and filter design," Systems \& Control Letters, vol. 60, pp. 665-673, 2011. [Online]. Available: http://dx.doi.org/10.1016/j. sysconle.2011.05.004

[23] W. J. Rugh, Linear System Theory. Prentice-Hall, 1996.

[24] T. Cipra and R. Romera, "Kalman filter with outliers and missing observations," Test, vol. 6, no. 2, pp. 379-395, 1997. [Online]. Available: http://dx.doi.org/10.1007/BF02564705

[25] Z. Đurović and B. Kovačević, "Robust estimation with unknown noise statistics," Automatic Control, IEEE Transactions on, vol. 44, no. 6, pp. 1292-1296, 1999. [Online]. Available: http://dx.doi.org/10. $1109 / 9.769393$

[26] S. Chan, Z. Zhang, and K. Tse, "A new robust Kalman filter algorithm under outliers and system uncertainties," in Circuits and Systems, 2005. ISCAS 2005. IEEE International Symposium on. IEEE, 2005, pp. 4317-4320. [Online]. Available: http://dx.doi.org/10.1109/ISCAS.2005.1465586

[27] P. J. Huber, Robust statistics. Wiley, New York, 1981.

[28] J.-A. Ting, E. Theodorou, and S. Schaal, "A Kalman filter for robust outlier detection," in Intelligent Robots and Systems, 2007. IROS 2007. IEEE/RSJ International Conference on. IEEE, 2007, pp. 1514-1519. [Online]. Available: http://dx.doi.org/10.1109/IROS.2007.4399158

[29] G. Agamennoni, J. I. Nieto, and E. M. Nebot, "An outlier-robust Kalman filter," in Robotics and Automation (ICRA), 2011 IEEE International Conference on. IEEE, May 2011, pp. 1551-1558. [Online]. Available: http://dx.doi.org/10.1109/ICRA.2011.5979605 